\title{
CONCERNING A THEOREM OF L. K. HUA AND I. REINER
}

\section{PETER STANEK}

1. Denote by $\operatorname{Sp}(2 n)$ the group of all $2 n$ by $2 n$ matrices of rational integers which satisfy

$$
\text { (1) } X H X^{T}=H, \quad H=\left(\begin{array}{cc}
0 & I \\
-I & 0
\end{array}\right), \quad X^{T}=X \text { transpose. }
$$

This is the symplectic modular group and in [1] Hua and Reiner show that $\operatorname{Sp}(2 n)$ may be generated by two matrices for $n=1$, and by four matrices for $n>1$. In this paper we improve their result and prove

Theorem. $\operatorname{Sp}(2 n)$ is generated by three matrices for $n=2$ and $n=3$, and by two matrices for $n>3$.

2. We define the following types of symplectic matrices:

(i) rotations

$$
\left(\begin{array}{cc}
A^{T} & 0 \\
0 & A^{-1}
\end{array}\right), \quad \operatorname{det} A= \pm 1,
$$

(ii) translations

$$
\left(\begin{array}{ll}
I & S \\
0 & I
\end{array}\right), \quad S^{T}=S
$$

(iii) semi-involutions

$$
\left(\begin{array}{cc}
Q & I-Q \\
Q-I & Q
\end{array}\right),
$$

where $Q$ is a diagonal matrix of zeros and ones. Then [1] $\operatorname{Sp}(2 n)$ is generated by the set of rotations, translations, and semi-involutions. Let $E_{i j}$ be the $n$ by $n$ matrix, all zero except for a one in the $i j$ th entry. Let $R_{i j}(x)$ be the rotation, as above, with $A=I+x E_{j i}$, for $i \neq j ; T_{i}(x)$ the translation with $S=x E_{i i}$; and $T_{i j}(x)$ the translation with $S=x E_{i j}+x E_{j i}$. Then the $T$ 's commute and

$$
\begin{aligned}
\left(T_{i}(x)\right)^{ \pm k} & =T_{i}( \pm k x), \\
\left(T_{i j}(x)\right)^{ \pm k} & =T_{i j}( \pm k x), \quad k \text { any integer. }
\end{aligned}
$$

If we let $(U, V)$ be the commutator, $U V U^{-1} V^{-1}$, then

Received by the editors August 17, 1962. 
(3)

$$
\begin{aligned}
\left(R_{i j}(x)\right)^{ \pm k} & =R_{i j}( \pm k x), & & k \text { any integer, } \\
\left(R_{i j}(x), R_{j \rho}(y)\right) & =R_{i \rho}(x y), & & i \neq \rho .
\end{aligned}
$$

Now, for $n>3, \operatorname{Sp}(2 n)$ is generated by $J=R_{21}(1) T_{n}(1)$ and $D$ :

$$
D=\left(\begin{array}{cc}
\sum_{i=1}^{n-1} E_{i, i+1} & -E_{n 1} \\
E_{n 1} & \sum_{i=1}^{n-1} E_{i, i+1}
\end{array}\right) .
$$

We compute

$$
\left(J, D^{-1} J D\right)=R_{31}(-1) .
$$

For indices $i$ and $j, n \geqq i>j \geqq 1$,

(8) $\quad D^{-k}\left(T_{j+n-i+1,1}(x)\right)^{T} D^{k}=\left(T_{j+n-i+1+k, 1+k}(x)\right)^{T}, \quad 0 \leqq k \leqq i-1-j$;

(9) $\quad D^{-1}\left(T_{n, i-j}(x)\right)^{T} D=R_{1, i-j+1}(-x)$;

(10) $\quad D^{-k} R_{1, i-j+1}(-x) D^{k}=R_{1+k, i-j+1+k}(-x), 0 \leqq k \leqq n+j-i-1$;

(11) $\quad D^{-1} R_{n+j-i, n}(-x) D=T_{n+j-i+1,1}(-x)$;

(12) $D^{-k} T_{n+j-i+1,1}(-x) D^{k}=T_{n+j-i+1+k, 1+k}(-x), \quad 0 \leqq k \leqq i-j-1$;

$$
D^{-1} T_{n, i-j}(-x) D=R_{i-j+1,1}(x)
$$

and

$$
D^{1-i} R_{i-j+1,1}(x) D^{i-1}=R_{i j}(x) .
$$

Hence $R_{13}(1)$ is obtained from $D$ and $J$.

$$
\left(J, R_{13}(1)\right)=R_{23}(1) \text {. }
$$

Equations (6)-(14) show $D$ and $J$ generate every $R_{i, i+1}(1), R_{i+1, i}(1)$. Repeated use of (3) and (4) will show every $R_{i j}(k), i \neq j, k$ any integer is obtained. Hence, the group generated by $D$ and $J$ contains every rotation, as above, with $\operatorname{det} A=1$.

$$
J R_{21}(-1)=T_{n}(1)
$$

and

$$
\begin{aligned}
D^{n-1} T_{n}(1) D^{1-n} & =T_{1}(1) . \\
D^{-1} T_{n}(1) D & =\left(T_{1}(-1)\right)^{T}=P .
\end{aligned}
$$




$$
T_{1}(1) P T_{1}(1)=\left(\begin{array}{cc}
I-E_{11} & E_{11} \\
-E_{11} & I-E_{11}
\end{array}\right)=S_{1}
$$

But $S_{1}^{2}$ is a rotation with $A=I-2 E_{11}$. Therefore, from $D$ and $J$ any rotation may be had. If we let $S_{i, j, k}, \ldots$ be the semi-involution where $Q$ has zeros in the $i i$ th, $j j$ th, $k k$ th, $\cdots$, positions and ones in the other diagonal positions, then

$$
\left(S_{i, j, k}, \ldots\right)\left(S_{i_{1}, j_{1}, k_{1}}, \ldots\right)=S_{i, j, k}, \cdots, i_{1}, j_{1}, k_{1}, \cdots
$$

Since

$$
D^{-k} S_{1} D^{k}=S_{1+k}, \quad 0 \leqq k \leqq n-1,
$$

clearly all semi-involutions are available.

To obtain all translations,

$$
D^{-1} R_{1 n}(k) D=T_{12}(k) .
$$

Since

$$
\begin{aligned}
\left(\begin{array}{ll}
I & S \\
0 & I
\end{array}\right)\left(\begin{array}{cc}
I & S^{1} \\
0 & I
\end{array}\right) & =\left(\begin{array}{cc}
I & S+S^{1} \\
0 & I
\end{array}\right), \\
\left(\begin{array}{cc}
U & 0 \\
0 & \left(U^{T}\right)^{-1}
\end{array}\right)\left(\begin{array}{cc}
I & S \\
0 & I
\end{array}\right)\left(\begin{array}{cc}
U^{-1} & 0 \\
0 & U^{T}
\end{array}\right) & =\left(\begin{array}{cc}
I & U S U^{T} \\
0 & I
\end{array}\right),
\end{aligned}
$$

by simultaneously interchanging rows and corresponding columns of the symmetric matrices $k E_{11}$ and $k E_{12}+k E_{21}$ of $T_{1}(k)$ and $T_{12}(k)$, respectively, every translation is available. This completes the proof for $n>3$.

3. For $n=2$ or 3 , it is now easy to see that the matrices $D, R_{21}(1)$, and $T_{1}(1)$ will generate $\operatorname{Sp}(2 n)$.

\section{REFERENCE}

1. L. K. Hua and I. Reiner, Generation of the symplectic modular group, Trans. Amer. Math. Soc. 65 (1949), 415-426.

Institute FOR Defense ANALyses 